Tôhoku Math. Journ.

27 (1975) 487-496.

\title{
HARMONIC AND QUASIHARMONIC DEGENERACY OF RIEMANNIAN MANIFOLDS
}

\author{
Lung Ock Chung and Leo Sario
}

(Received May 7, 1974)

The harmonic and quasiharmonic classifications of Riemannian manifolds have been largely brought to completion (see Bibliography). In the present paper we shall discuss interrelations between harmonic null classes and quasiharmonic null classes.

Let $H$ and $Q$ be the classes of harmonic and quasiharmonic functions $h$ and $q$, defined by $\Delta h=0$ and $\Delta q=1$, where $\Delta=d \delta+\delta d$ is the LaplaceBeltrami operator. Denote by $P, B, D, C, L^{p}$ the classes of functions which are positive, bounded, Dirichlet finite, bounded Dirichlet finite, or of finite $L^{p}$ norm, respectively. Here $1 \leqq p<\infty$, the value $p=\infty$ being excluded since for both harmonic and quasiharmonic functions, $L^{\infty}=B$. For $X=P, B, D, C, L^{p}$, set $H X=H \cap X, Q X=Q \cap X$, and let $O_{F}^{N}$ stand for the class of Riemannian $N$-manifolds, $N \geqq 2$, which do not carry nonconstant functions in a given class $F$. The complement of $O_{F}^{N}$ with respect to the totality of Riemannian $N$-manifolds is designated by $\widetilde{O}_{F}^{N}$.

We shall first show that $O_{H X}^{N} \cap O_{Q Y}^{N} \neq \varnothing$ for $X, Y=P, B, D, C, L^{p}$, $1 \leqq p<\infty, N \geqq 2$. In view of the Euclidean ball it is trivial that $\widetilde{O}_{H X}^{N} \cap$ $\widetilde{O}_{Q Y}^{N} \neq \varnothing$, and we shall prove that $\widetilde{O}_{H X}^{N} \cap O_{Q Y}^{N} \neq \varnothing$ for all $X, Y, p, N$.

The classes $O_{H X}^{N} \cap \widetilde{O}_{Q Y}^{N}$ are intriguing. From the harmonic classification theory it is known that the class $O_{G}^{N}$ of parabolic $N$-manifolds, characterized by the nonexistence of Green's functions, is related to other harmonic null classes by the strict inclusion relations $O_{G}^{N}<O_{H P}^{N}<O_{H B}^{N}<$ $O_{H D}^{N}=O_{H C}^{N}$. The proof of the strictness, due mainly to Ahlfors, Royden, and Tôki, was one of the most challenging problems in the theory of harmonic functions. On the other hand, $O_{G}^{N}$ is strictly contained also in all $O_{Q X}^{N}, X=P, B, D, C[12,20]$. The problem of proving the nonemptiness of the classes $O_{H X}^{N} \cap \widetilde{O}_{Q Y}^{N}$ thus amounts to finding manifolds which belong to the "narrow" spaces $\widetilde{O}_{G}^{N} \cap O_{H X}^{N}$, yet carry $Q Y$-functions. For $X, Y$ other than $L^{p}$ we only have fragmentary results on this problem (see No. 11). On the other hand, the classes $O_{H X}^{N} \cap \widetilde{O}_{Q Y}^{N}$ turn out to be

The work was sponsored by the U.S. Army Research Office, Grant DA-ARO-31-124-73G39, University of California, Los Angeles.

MOS Classification: 31B30. 
nonvoid for $X=L^{p}$, all $Y, p, N ; Y=L^{p}$, all $X, p, N$; and $X=L^{p}, Y=L^{q}$, all $p, q, N$.

1. Our first problem is to exclude both $H X$ and $Q Y$ functions:

Theorem 1. $O_{H X}^{N} \cap O_{Q T}^{N} \neq \varnothing$ for $X, Y=P, B, D, C, L^{p} ; 1 \leqq p<\infty$; $N \geqq 2$.

The proof will be given in Nos. 2-4.

2. By means of the Poisson integral and Harnack's inequality we see immediately that every $h \in H P$ in the Euclidean $N$-space $E^{N}$ reduces to a constant. Therefore $E^{N} \in O_{H X}^{N}$ for $X=P, B, D, C$.

To show that $E^{N} \in O_{H L^{p}}^{N}$ we first consider the case $p=1$. Suppose there exists a nonconstant $h \in H L^{1}$. It has a unique representation $h=$ $\sum_{n=0}^{\infty} r^{n} S_{n}$, with $S_{n}=S_{n}(\theta)$ spherical harmonics, characterized by $\Delta\left(r^{n} S_{n}\right)=$ 0 , and $(r, \theta)=\left(r, \theta_{1}, \cdots, \theta_{N-1}\right)$ the polar coordinates. For some $n_{0}>0$, $S_{n_{0}} \not \equiv 0$. Take a function $\rho(r) \in C[0, \infty)$ with $\rho(r)=1 / r$ for $r \geqq 1$ and set $\varphi=\rho S_{n_{0}}$. Since $\varphi \in B$, we have $\|h \varphi\|_{1}<\infty$. On the other hand,

$$
\begin{aligned}
\|h \varphi\|_{1} & \geqq\left|\left(h \rho, S_{n_{0}}\right)\right|=c \int_{0}^{\infty} r^{n_{0}} \rho r^{N-1} d r \\
& =a+c \int_{1}^{\infty} r^{n_{0}+N-2} d r=\infty ;
\end{aligned}
$$

here and later $c$ is a constant, not always the same. The contradiction shows that $E^{N} \in O_{H L^{1}}^{N}$.

Now let $p>1$ and take $p^{\prime}$ with $1 / p+1 / p^{\prime}=1$. Suppose there exists a nonconstant $h \in H L^{p}$. In the expansion $h=\sum_{0}^{\infty} r^{n} S_{n}, S_{n_{0}} \not \equiv 0$ for some $n_{0}>0$. Let $\rho(r) \in C[0, \infty)$ with

$$
\rho(r)=r^{-(N+1) / p^{\prime}} \text { for } r \geqq 1,
$$

and set $\varphi=\rho S_{n_{0}}$. Since

$$
\|\rho\|_{p^{\prime}}^{p^{\prime}}=a+c \int_{1}^{\infty} r^{-(N+1)} r^{N-1} d r<\infty,
$$

$\|\varphi\|_{p^{\prime}}<\infty$, and $|(h, \varphi)|<\infty$. We again have a contradiction:

$$
\begin{aligned}
|(h, \varphi)| & =\left|\mathrm{a}+\mathrm{c} \int_{1}^{\infty} r^{n_{0}} r^{-(N+1) / p^{\prime}} r^{N-1} d r\right| \\
& =\left|a+c \int_{1}^{\infty} r^{n_{0}+N / p-1 / p^{\prime}-1} d r\right|=\infty .
\end{aligned}
$$

A fortiori $E^{N} \in O_{H L^{p}}^{N}$ for $p>1$ as well.

3. To prove $E^{N} \in O_{Q X}^{N}$ for $X=P, B, D, C$ we recall that $O_{Q P}^{N}<O_{Q B}^{N} \cap$ 
$O_{Q D}^{N}<O_{Q B}^{N} \cup O_{Q D}^{N}=O_{Q C}^{N}[12,20]$. Thus it suffices to establish $E^{N} \in O_{Q P}^{N}$. Since

$$
q_{0}=-(2 N)^{-1} r^{2} \in Q,
$$

every $q \in Q$ can be written $q=q_{0}+h$ with some $h \in H$. We are to show that $q \notin P$. Set

$$
q=q_{0}+h(0)+k, \quad k \in H, \quad k(0)=0,
$$

where $h(0), k(0)$ are the values at the origin. By the mean value theorem there exists, for every $r_{n}$, an $\theta^{n}=\left(\theta_{1}^{n}, \cdots, \theta_{N-1}^{n}\right)$ such that $k\left(r_{n}, \theta^{n}\right)=0$. If $\left\{r_{n}\right\}_{0}^{\infty}$ is an increasing sequence with $r_{n} \rightarrow \infty$, then

$$
q\left(r_{n}, \theta^{n}\right)=-\frac{1}{2 N} r_{n}^{2}+h(0) \rightarrow-\infty,
$$

and therefore $q \notin P$.

4. It remains to show that $E^{N} \in O_{Q L}^{N}$. Again we start with $p=1$. For $q \in Q, q=q_{0}+h(0)+k$, we have

$$
k(0)=\int_{E^{N}} k d V=0,
$$

and therefore

$$
\|q\|_{1} \geqq\left|\int_{E^{N}}\left(q_{0}+h(0)\right) d V\right| .
$$

The integrand (with respect to $d r d \theta_{1} \cdots d \theta_{N-1}$ ) is $\sim c r^{2} r^{N-1}$, hence $\|q\|_{1}=\infty$, and $E^{N} \in O_{Q L^{1}}^{N}$.

In the case $p>1$, the choice $\varphi(r) \in C[0, \infty), \varphi(r)=r^{-(N+1) / p^{\prime}}$ for $r \geqq 1$ gives $\|\varphi\|_{p^{\prime}}<\infty$. If there exists a $q \in Q L^{p}, q=q_{0}+h(0)+k$, then by $(k, \varphi)=0$, the integrand in $(q, \varphi)=\left(q_{0}+h(0), \varphi\right)$ is asymptotically

$$
c \boldsymbol{r}^{2} \boldsymbol{r}^{-(N+1) / p^{\prime}} \boldsymbol{r}^{N-1}=\boldsymbol{c} \boldsymbol{r}^{N / p-1 / p^{\prime}+1} \text {. }
$$

The exponent dominates $N / p$, hence $|(q, \varphi)|=\infty$, in violation of $\|\varphi\|_{p^{\prime}}<\infty$.

The proof of Theorem 1 is herewith complete.

5. In view of the Euclidean $N$-ball it is trivial that $\widetilde{O}_{H X}^{N} \cap \widetilde{O}_{Q Y}^{N} \neq \varnothing$ for $X, Y=P, B, D, C, L^{p} ; 1 \leqq p<\infty ; N \geqq 2$. Our next problem is to find an $N$-manifold which carries $H X$ functions but no $Q Y$ functions.

THEOREM 2. $\widetilde{O}_{H X}^{N} \cap O_{Q T}^{N} \neq \varnothing$ for $X, Y=P, B, C, D, L^{p} ; 1 \leqq p<\infty$; $N \geqq 2$.

The proof will be given in Nos. 6-10.

6. For $X, Y=P, B, D, C$ it suffices to show that $\widetilde{O}_{H D}^{N} \cap O_{Q P}^{N} \neq \varnothing$. 
Consider the "beam"

$$
T=\left\{\left(x, y_{1}, \cdots, y_{N-1}\right)|| x|<\infty,| y_{i} \mid \leqq 1, i=1, \cdots, N-1\right\},
$$

with each face $y_{i}=1$ identified with the opposite face $y_{i}=-1$ by a parallel translation perpendicular to the $x$-axis. Endow $T$ with a metric with volume element $\sqrt{g} d x d y_{1} \ldots d y_{N-1}$, where $g$ is the determinant of the metric tensor $\left(g_{i j}\right)$ with inverse $\left(g^{i j}\right)$. Let each $g_{i j}$ depend on $x$ only and set

$$
\rho(x)=\sqrt{g(x)}, \quad \sigma(x)=g^{11}(x) .
$$

Then $h_{0}(x)$ is harmonic if and only if

$$
\Delta h_{0}=-\rho^{-1}\left(\rho \sigma h_{0}^{\prime}\right)^{\prime}=0,
$$

that is,

$$
h_{0}(x)=c \int_{a}^{x} \rho^{-1} \sigma^{-1} d t .
$$

The Dirichlet integral over the subspace from $-x$ to $x$ is

$$
D_{x}\left(h_{0}\right)=c \int_{-x}^{x} h_{0}^{\prime 2} \sigma \rho d t=c \int_{-x}^{x} \rho^{-1} \sigma^{-1} d t .
$$

We recall (loc. cit.) that a manifold belongs to $\widetilde{O}_{Q P}^{N}$ if and only if the potential $G 1$ of the constant function 1,

$$
G 1(\xi)=\int_{\eta \in T} g(\xi, \eta) d V(\eta)
$$

exists at some, and hence every, $\xi \in T$. In the present case this potential depends on the $x$-coordinate $x(\xi)$ of $\xi$ only, and we can use the notation $G 1(x)$. Suppose $G 1(x)$ exists. Since $\Delta G 1(x) \equiv 1$,

$$
G 1(x)=q_{0}(x)+h_{0}(x),
$$

where

$$
q_{0}(x)=-\int_{0}^{x} \rho^{-1} \sigma^{-1} \int_{0}^{t} \rho d s d t
$$

is quasiharmonic by $\Delta q_{0}=-\rho^{-1}\left(\rho \sigma q_{0}^{\prime}\right)^{\prime}=1$. Our problem thus reduces to finding functions $\rho(x), \sigma(x)$ such that

$$
\begin{gathered}
\int_{-x}^{x} \rho^{-1} \sigma^{-1} d t \in B, \\
\int_{0}^{x} \rho^{-1} \sigma^{-1} \int_{0}^{t} \rho(s) d s d t \rightarrow \infty \quad \text { as } \quad x \rightarrow \infty .
\end{gathered}
$$

The choice $\rho=1, \sigma=1+x^{2}$ gives 


$$
\int_{0}^{x} \rho^{-1} \sigma^{-1} d t=\int_{0}^{x}\left(1+t^{2}\right)^{-1} d t=\arctan x \in H D,
$$

hence $T \in \widetilde{O}_{H D}^{N}$. An arbitrary $h_{0}(x) \in H$ is

$$
h_{0}(x)=a \arctan x+b,
$$

and

$$
q_{0}(x)=-\int_{0}^{x}\left(1+t^{2}\right)^{-1} t d t=-\frac{1}{2} \log \left(1+x^{2}\right),
$$

which tends to $-\infty$ as $|x| \rightarrow \infty$. Therefore $G 1=q_{0}+h_{0} \rightarrow-\infty, G 1 \notin P$, and consequently $T \in O_{Q P}^{N}$.

The relation $\widetilde{O}_{H D}^{N} \cap O_{Q P}^{N}$ we have thus established can be generalized to polyharmonic functions [3].

7, The relation

$$
\widetilde{O}_{H L^{p}}^{N} \cap O_{Q Y}^{N} \neq \varnothing
$$

was proved in [1] for $Y=P, B, D, C ; 1 \leqq p<\infty ; N \geqq 2$. We proceed to show that

$$
\widetilde{O}_{H X}^{N} \cap O_{Q L^{p}}^{N} \neq \varnothing
$$

for $X=P, B, D, C ; 1 \leqq p<\infty ; N \geqq 2$. Consider the manifold

$$
T:\left\{\left(x, y_{1}, \cdots, y_{N-1}\right)|x>1,| y_{i} \mid \leqq \pi, i=1, \cdots, N-1\right\} \text {. }
$$

It shall be henceforth tacitly understood that the opposite faces $y_{i}=\pi$ and $y_{i}=-\pi$ of such "beams" are identified by pairs as in No. 6. For the metric we choose

$$
d s^{2}=d x^{2}+x^{2 \alpha /(N-1)} \sum_{i=1}^{N-1} d y_{i}^{2}
$$

with $\alpha$ a constant $>1$.

To show that $T \in \widetilde{O}_{H X}^{N}$ it suffices to take $X=C$. The function $x^{-\alpha+1}$ is in $H B$, by virtue of

$$
\Delta x^{-\alpha+1}=-x^{-\alpha}\left(x^{\alpha}\left(x^{-\alpha+1}\right)^{\prime}\right)^{\prime}=0 .
$$

Moreover,

$$
D\left(x^{-\alpha+1}\right)=c \int_{1}^{\infty}\left(x^{-\alpha}\right)^{2} x^{\alpha} d x<\infty
$$

Therefore $T \in \widetilde{O}_{H C}^{N}$.

8. To prove $T \in O_{Q L^{p}}^{N}$ we first take $p=1$. Since

$$
q_{0}(x)=-\frac{1}{2}(\alpha+1)^{-1} x^{2} \in Q,
$$


every $q \in Q$ can be written

$$
q=q_{0}+a x^{-\alpha+1}+b+\Sigma^{\prime} f_{n}(x) G_{n}(y),
$$

where $f_{n} G_{n} \in H$ and $G_{n}$ ranges over all products of the form

$$
G_{n}(y)=\prod_{i=1}^{N-1} \sin n_{i} y_{i}
$$

with $n=\left(n_{1}, \cdots, n_{N-1}\right) \neq(0, \cdots, 0)$. Since $\int_{T} f_{n} G_{n} d V=0$,

$$
\|q\|_{1} \geqq\left|\int_{T}\left(q_{0}+a x^{-\alpha+1}+b\right) d V\right| .
$$

The integrand is $\sim c x^{2+\alpha}$, hence $\|q\|_{1}=\infty$ and $T \in O_{Q L^{1}}^{N}$.

Now let $p>1$, and $p^{\prime}$ as before. The function $\phi(x)=x^{-\left(\alpha+p^{\prime}\right) / p^{\prime}}$ belongs to $L^{p^{\prime}}$ by virtue of

$$
\int_{T}|\varphi|^{p^{\prime}} d V=c \int_{1}^{\infty} x^{-\left(\alpha+p^{\prime}\right)} x^{\alpha} d x<\infty .
$$

If there exists a $q \in L^{p}$, then $|(q, \varphi)|<\infty$. On the other hand,

$$
|(q, \varphi)|=\left|\int_{T}\left(q_{0}+a x^{-\alpha+1}+b\right) x^{-\left(\alpha+p^{\prime}\right) / p^{\prime}} d V\right|,
$$

where the integrand with respect to $d x d y_{1} \cdots d y_{N-1}$ is asymptotically $x^{\alpha / p+1}$. A fortiori $|(q, \varphi)|=\infty$, and the contradiction gives $T \in O_{Q L^{p}}^{N}$.

9. It remains to show that

$$
\widetilde{O}_{H L^{s}}^{N} \cap O_{Q L^{t}}^{N} \neq \varnothing
$$

for $1 \leqq s<\infty, 1 \leqq t<\infty$. First fix $t$ and consider the manifold

$$
T=\left\{\left(x, y_{1}, \cdots, y_{N-1}\right)|x>1,| y_{i} \mid \leqq \pi, i=1, \cdots, N-1\right\}
$$

with the metric

$$
d s^{2}=e^{-x / t} d x^{2}+e^{(2 x+x / t) /(N-1)} \sum_{\imath=1}^{N-1} d y_{\imath}^{2} .
$$

The function $h_{0}(x)=e^{-x-x / t}$ belongs to $H L^{s}$, and $T \in \widetilde{O}_{H L^{s}}^{N}$.

10. To see that $T \in O_{Q L^{t}}^{N}$, note that $q_{0}(x)=t e^{-x / t} \in Q$. Every $q \in Q$ has the form

$$
q=q_{0}+a h_{0}+b+\Sigma^{\prime} f_{n} G_{n}
$$

For $t=1$,

$$
\|q\|_{1} \geqq c\left|\int_{1}^{\infty}\left(q_{0}+a h_{0}+b\right) e^{x} d x\right|
$$


If $b \neq 0$, then the integrand is $\sim b e^{x}$, and $\|q\|_{1}=\infty$. If $b=0$, the dominating term in the integrand is $q_{0} e^{x}$, so that again $\|q\|_{1}=\infty$, and $T \in O_{Q L^{1}}^{N}$.

In the case $t>1$, take $t^{\prime}$ such that $1 / t+1 / t^{\prime}=1$. The function $\varphi(x)=e^{-x / t^{\prime}} x^{-1}$ belongs to $L^{t^{\prime}}$. If there exists a $q \in Q L^{t}$, then $|(q, \varphi)|<\infty$. But

$$
|(q, \varphi)|=\left|\left(q_{0}+a h_{0}+b+\Sigma^{\prime} f_{n} G_{n}, \varphi\right)\right|=\left|\left(q_{0}+a h_{0}+b, \varphi\right)\right| .
$$

If $b \neq 0$, the integrand is $\sim b e^{-x / t^{\prime}} x^{-1} e^{x}=b e^{x / t} x^{-1}$, and we have the contradiction $|(q, \varphi)|=\infty$. If $b=0$, since $q_{0}$ dominates $h_{0}$, the integrand is asymptotically

$$
c e^{-x / t-x / t^{\prime}} x^{-1} e^{x}=c x^{-1},
$$

and again we have divergence. Therefore $T \in O_{Q L^{t}}^{N}$.

The proof of Theorem 2 is herewith complete.

11. Our final problem is to find $N$-manifolds which carry $Q Y$ functions but no $H X$ functions. For $X, Y$ other than $L^{p}$ we only have two fragmentary results, both quite immediate. First, $O_{H D}^{N} \cap \widetilde{O}_{Q C}^{N} \neq \varnothing$ for $N \geqq 5$ is a consequence of what is known of the Poincare $N$-ball $B_{\alpha}^{N}$, that is, the ball $\{r<1\}$ with the metric $d s=\left(1-r^{2}\right)^{\alpha}|d x|, \alpha$ a real constant. It was shown in [5] that $B_{\alpha}^{N} \in O_{H D}^{N}$ if and only if $|\alpha| \geqq(N-2)^{-1}$, $N \geqq 3$, and in [26] that

$$
B_{\alpha}^{N} \in \widetilde{O}_{Q B}^{N} \cap \widetilde{O}_{Q D}^{N}=\widetilde{O}_{Q C}^{N}
$$

if and only if $-3 /(N+2)<\alpha<1 /(N-2), N \geqq 3$. A fortiori,

$$
B_{\alpha}^{N} \in O_{H D}^{N} \cap \widetilde{O}_{Q C}^{N}, \quad N \geqq 5,
$$

if and only if $-3 /(N+2)<\alpha \leqq-1 /(N-2)$.

Another result, $O_{H D}^{N} \cap \widetilde{O}_{Q B}^{N} \neq \varnothing$ and $O_{H D}^{N} \cap \widetilde{O}_{Q P}^{N} \neq \varnothing$, both for $N \geqq 4$, is also offered by $B_{\alpha}^{N}$. We know [26] that $B_{\alpha}^{N} \in \widetilde{O}_{Q B}^{N}$ if and only if $-1<$ $\alpha<1 /(N-2)$, and $B_{\alpha}^{N} \in \widetilde{O}_{Q P}^{N}$ is characterized by the same range of $\alpha$. As a consequence,

$$
B_{\alpha}^{N} \in O_{H D}^{N} \cap \widetilde{O}_{Q B}^{N}\left(\widetilde{O}_{Q P}^{N}\right), \quad N \geqq 4,
$$

if and only if $-1<\alpha \leqq-1 /(N-2)$.

12. In the case of $L^{p}$ functions we have a complete result:

THEOREM 3. $O_{H X}^{N} \cap \widetilde{O}_{Q Y}^{N} \neq \varnothing$ for $X=L^{p}, Y=P, B, D, C, 1 \leqq p<\infty$, $N \geqq 2 ; X=P, B, D, C, Y=L^{p}, 1 \leqq p<\infty, N \geqq 2$; and $X=L^{s}, Y=L^{t}$, $1 \leqq s<\infty, 1 \leqq t<\infty, N \geqq 2$.

The proof will be given in Nos. 13-14. 
13. The first case,

$$
O_{H L^{p}}^{N} \cap \widetilde{O}_{Q T}^{N} \neq \varnothing
$$

for $Y=P, B, D, C, 1 \leqq p<\infty, N \geqq 2$, was established in [1], and we proceed to the case $O_{H X}^{N} \cap \widetilde{O}_{Q L}^{N} \neq \varnothing$ for $X=P, B, D, C, 1 \leqq p<\infty, N \geqq 2$. It suffices to prove

$$
O_{H P}^{N} \cap \widetilde{O}_{Q L^{p}}^{N} \neq \varnothing
$$

Take the manifold

$$
T=\left\{\left(x, y_{1}, \cdots, y_{N-1}\right)|| x|<\infty,| y_{i} \mid \leqq 1, i=1, \cdots, N-1\right\}
$$

with the metric

$$
d s^{2}=e^{-x^{2}} d x^{2}+e^{-x^{2} /(N-1)} \sum_{i=1}^{N-1} d y_{i}^{2}
$$

Since $x \in H, T$ is parabolic, hence in $O_{H P}^{N}$. The function

$$
q_{0}(x)=-\int_{0}^{x} \int_{0}^{t} e^{-s^{2}} d s d t
$$

is quasiharmonic and for $1 \leqq p<\infty$

$$
\left\|q_{0}\right\|_{p}^{p}=c \int_{-\infty}^{\infty}\left|q_{0}\right|^{p} e^{-x^{2}} d x<\infty \text {. }
$$

In fact, $\left|q_{0}\right|^{p} \leqq\left|\int_{0}^{x} a d x\right|^{p} \leqq a^{p}|x|^{p}$, where $a=\int_{0}^{\infty} e^{-t^{2}} d t$. Therefore $T \in O_{Q_{L} p}^{N}$.

14. The remaining case of Theorem 3 is

$$
O_{H L^{s}}^{N} \cap \widetilde{O}_{Q L^{t}}^{N} \neq \varnothing
$$

for $1 \leqq s<\infty, 1 \leqq t<\infty, N \geqq 2$. Take the $N$-space $M$ with the metric

$$
d s^{2}=\varphi(r) d r^{2}+\psi(r)^{1 /(N-1)} \sum_{i=1}^{N-1} \lambda_{i}(\theta) d \theta_{i}^{2},
$$

where $\varphi, \psi \in C[0, \infty)$ and the $\lambda_{i}$ are trigonometric functions of $\theta=$ $\left(\theta_{1}, \cdots, \theta_{N-1}\right)$ such that the metric is Euclidean for $r \leqq 1 / 2$. For $r \geqq 1$ we choose

$$
\varphi(r)=e^{-2 r}, \quad \psi(r)=e^{2 r} .
$$

The volume element of $M$ for $r \geqq 1$ is simply $d r d \theta_{1} \cdots d \theta_{N-1}$.

To prove $M \in O_{H L^{s}}^{N}$ take a nonconstant

$$
h \in H, \quad h=\Sigma f_{n}(r) S_{n}(\theta) .
$$

In each term, if $f_{n} \not \equiv 0$, then by the maximum principle, $f_{n}(r) \neq 0$ for every $r>0$, and $\lim _{r \rightarrow \infty} f_{n}(r) \neq 0$, so that $\left|f_{n}(r)\right|>c_{n}>0$ for $r>1$, say. 
For some $n_{0}, f_{n_{0}} \not \equiv 0$. Take a function $\rho(r) \in C[0, \infty)$ with $\rho(r)=1 / r$ for $r \geqq 1$, and set $\varphi=\rho S_{n_{0}}$. Since $\|\rho\|_{\boldsymbol{s}^{\prime}}<\infty,\|\varphi\|_{\boldsymbol{s}^{\prime}}<\infty$. If $h \in L^{s}$, then $|(h, \varphi)|<\infty$. But

$$
|(h, \varphi)|=\left|a+b \int_{1}^{\infty} f_{n_{0}} \rho d r\right|,
$$

where $\left|f_{n_{0}} \rho\right| \geqq c_{n_{0}} / r$ and $f_{n_{0}}$ is of constant sign, hence $|(h, \varphi)|=\infty$. This contradiction gives $M \in O_{H L}^{N}$.

To see that $M \in \widetilde{O}_{Q L}^{N}, 1 \leqq t<\infty$, we note that the function

$$
q_{0}(r)=\int_{r}^{\infty} \varphi^{1 / 2} \psi^{-1 / 2} \int_{0}^{t}(\varphi \psi)^{1 / 2} d s d t
$$

is quasiharmonic and $\left\|q_{0}\right\|_{t}<\infty$.

This completes the proof of Theorem 3.

The authors are indebted to Professor Cecilia Wang for a careful checking of the manuscript.

\section{BIBLIOGRAPHY}

[1] L. ChUng AND L. SARIo, Harmonic $L^{p}$ functions and quasiharmonic degeneracy, J. Indian Math. Soc., (to appear).

[2] L. Chung, L. SARio AND C. WANG, Quasiharmonic $L^{p}$-functions on Riemannian manifolds, Ann. Scuola Norm. Sup. Pisa, (to appear).

[3] L. Chung, L. Sario and C. Wang, Riemannian manifolds with bounded Dirichlet finite polyharmonic functions, Ann. Scuola Norm. Sup. Pisa, 27 (1973), 1-6.

[4] D. HAda, L. SARIo aNd C. Wang, Dirichlet finite biharmonic functions on the Poincaré $N$-ball, J. Reine Angew. Math., 272 (1975), 92-101.

[5] D. HADA, L. SARIO AND C. WANG, $N$-manifolds carrying bounded but no Dirichlet finite harmonic functions, Nagoya Math. J., 54 (1974), 1-6.

[6] O. HAUPT, Uber das asymptotische Verhalten der Lösungen gewisser linearer gewöhnlicher Differentialgleichungen, Math. Z., 48 (1913), 289-292.

[7] E. Hille, Behavior of solutions of linear second order differential equations, Ark. Mat., 2 (1952), 25-41.

[8] Y. K. Kwon, L. Sario and B. Walsh, Behavior of biharmonic functions on Wiener's and Royden's compactifications, Ann. Inst. Fourier (Grenoble), 21 (1971), 217-226.

[9] N. MirSKY, L. SARIO AND C. WANG, Bounded polyharmonic functions and the dimension of the manifold, J. Math. Kyoto Univ., 13 (1973), 529-535.

[10] M. NARAI AND L. SARIo, Completeness and function-theoretic degeneracy of Riemannian spaces, Proc. Nat. Acad. Sci., 57 (1967), 29-31.

[11] M. NakaI aNd L. SaRio, Biharmonic classification of Riemannian manifolds, Bull. Amer. Math. Soc., 77 (1971), 432-436.

[12] M. Nakai and L. Sario, Quasiharmonic classification of Riemannian manifolds, Proc. Amer. Math. Soc., 31 (1972), 165-169.

[13] M. NAKaI and L. SaRio, Dirichlet finite biharmonic functions with Dirichlet finite Laplacians, Math. Z., 122 (1971), 203-216.

[14] M. NAKaI aNd L. SaRio, A property of biharmonic functions with Dirichlet finite Laplacians, Math. Scand., 29 (1971), 307-316. 
[15] M. Nakai ANd L. Sario, Existence of Dirichlet finite biharmonic functions, Ann. Acad. Sci. Fenn. A. I, 532 (1973), 1-33.

[16] M. NaKaI and L. SaRio, Existence of bounded biharmonic functions, J. Reine Angew. Math. 259 (1973), 147-156.

[17] M. NAKaI AND L. SARIo, Existence of bounded Dirichlet finite biharmonic functions, Ann. Acad. Sci. Fenn. A. I, 505 (1972), 1-12.

[18] M. NAKai AND L. SARIo, Biharmonic functions on Riemannian manifolds, Continuum Mechanics and Related Problems of Analysis, Nauka, Moscow, 1972, 329-335.

[19] L. SARIO, Biharmonic and quasiharmonic functions on Riemannian manifolds, Duplicated lecture notes 1968-70, University of California, Los Angeles.

[20] L. SARIo, Quasiharmonic degeneracy of Riemannian $N$-manifolds, Ködai Math. Sem. Rep., 26 (1974), 53-57.

[21] L. SARIo, Completeness and existence of bounded biharmonic functions on a Riemannian manifold, Ann. Inst. Fourier (Grenoble), 24 (1974), 311-317.

[22] L. SARIO AND M. NAKAI, Classification Theory of Riemann Surfaces, Springer-Verlag, 1970 , pp. 446.

[23] L. Sario and C. WaNg, The class of $(p, q)$-biharmonic functions, Pacific J. Math., 41 (1972), 799-808.

[24] L. SARIO AND C. WANG, Counterexamples in the biharmonic classification of Riemannian 2-manifolds, Pacific J. Math., 50 (1974), 159-162.

[25] L. SARIO AND C. WANG, Generators of the space of bounded biharmonic functions, Math. Z., 127 (1972), 273-280.

[26] L. Sario and C. WaNG, Quasiharmonic functions on the Poincaré $N$-ball, Rend. Mat., 6 (1974), 1-14.

[27] L. Sario aNd C. WaNG, Riemannian manifolds of dimension $N \geqq 4$ without bounded biharmonic functions, J. London Math. Soc., 7 (1974), 635-644.

[28] L. SARIO AND C. WANG, Existence of Dirichlet finite biharmonic functions on the Poincaré 3-ball, Pacific J. Math., 48 (1973), 267-274.

[29] L. Sario and C. WaNG, Negative quasiharmonic functions, Tôhoku Math. J., 26 (1974), 85-93.

[30] L. Sario and C. Wang, Radial quasiharmonic functions, Pacific J. Math., 46 (1973), 515-522.

[31] L. SARIO AND C. WANG, Parabolicity and existence of bounded biharmonic functions, Comm. Math. Helv., 47 (1972), 341-347.

[32] L. Sario aNd C. WaNG, Positive harmonic functions and biharmonic degeneracy, Bull. Amer. Math. Soc., 79 (1973), 182-187.

[33] L. Sario and C. Wang, Parabolicity and existence of Dirichlet finite biharmonic functions, J. London Math. Soc., 8 (1974), 145-148.

[34] L. SARIO AND C. WANG, Harmonic and biharmonic degeneracy, Kōdai Math. Sem Rep., 25 (1973), 392-396.

[35] L. SARIo AND C. WaNG, Harmonic $L^{p}$-functions on Riemannian manifolds, Kōdai Math. Sem. Rep., 26 (1975), 204-209.

[36] L. Sario, C. WANG AND M. RaNGe, Biharmonic projection and decomposition, Ann. Acad. Sci. Fenn. A. I, 494 (1971), 1-14.

[37] C. Wang AND L. Sario, Polyharmonic classification of Riemannian manifolds, J. Math. Kyoto Univ., 12 (1972), 129-140.

Department of Mathematics

UNIVERSITY OF CALIFORNIA

Los ANgeles 90024, California

U.S.A. 